\title{
Educación continua en enfermería: Campus Virtual en Salud Pública en la Región de las Américas*
}

\author{
Continuing Education in Nursing: Virtual Campus in Public Health in Americas Region
}

Educação continuada em enfermagem: Campus Virtual em Saúde Pública na Região das Américas

\section{Silvia Cassiani \\ Organización Panamericana de la Salud, Estados Unidos \\ ORCID: https://orcid.org/0000-0003-0914-7511}

Rita Tereza de Almeida ${ }^{\text {a }}$

Universidad de São Paulo, Brasil

ritatereza1@usp.br

ORCID: https://orcid.org/0000-0002-1440-0609

Maria Cristina Hoyos Garcia

Universidad de Antioquia, Colombia

ORCID: https://orcid.org/0000-0001-9579-2335

Gabriel Listovsky

OPS/OMS, Panamá

ORCID: https://orcid.org/0000-0002-6007-2124

Edgardo M de Gracia Tejada

Organización Panamericana de la Salud, Panamá

ORCID: https://orcid.org/0000-0003-0237-2513

Lilia Jannet Saldarriaga Sandoval

Universidad Nacional de Tumbes, Perú

ORCID: https://orcid.org/0000-0002-1773-6669

Fernando Antonio Menezes da Silva

Organización Panamericana de la Salud, Estados Unidos

ORCID: https://orcid.org/0000-0002-0007-3993
DOI: https://doi.org/10.11144/Javeriana.ie23.ecec

Recibido: 07 Diciembre 2020

Aceptado: 04 Febrero 2021

Publicado: 15 Septiembre 2021

\section{Resumen:}

Introducción: educación continua es una inversión personal y profesional necesaria a lo largo de la vida profesional de los enfermeros, porque amplía y perfecciona conocimientos técnicos, científicos y habilidades prácticas. Objetivo: describir la participación de enfermeros de la Región de las Américas en los cursos ofrecidos por el Campus Virtual de Salud Pública antes y al comienzo de la pandemia de la COVID-19, según género, edad, nivel educativo y lugar de trabajo. Método: estudio descriptivo cuantitativo. Se analizaron datos de los participantes de los cursos del periodo de enero del 2013 hasta junio del 2020 del Campus Virtual de Salud Pública. Población de estudio: enfermeros de la Región de las Américas que tomaron cursos virtuales. Se calcularon medidas de estadística descriptiva y proporciones de las variables sociodemográficas de los participantes de manera confidencial con autorización y credenciales para acceder datos en la plataforma. Resultados: a los 515 cursos virtuales ofrecidos por el Campus Virtual de Salud Pública se matricularon 368.018 enfermeros. Los porcentajes de enfermeros con certificado según el país de procedencia son los siguientes: Ecuador con 64,7\%, México con 58,3\%, Honduras con 52,9\%, Paraguay con $48,6 \%$, Colombia con $45,2 \%$, Uruguay con $42,2 \%$, Argentina con $38,0 \%$, Chile con $22,7 \%$, Perú con $21,3 \%$, y Brasil con 9,7\%. El $83,1 \%$ eran mujeres, el $75 \%$ tenía un nivel educativo universitario y el $40 \%$ tenía edades entre 26 y 35 años y el $47,1 \%$ trabajaba en hospitales. Conclusión: la pandemia de la COVID-19 refuerza la importancia de la educación continua virtual en la que los enfermeros pueden mejorar sus conocimientos, potencializar sus habilidades, y calificarse para los cambios en la práctica, la enseñanza y la investigación.

Palabras clave: enfermería, educación en enfermería, educación a distancia, educación continua.

Notas de autor

a Autora de correspondencia. Correo electrónico: ritatereza1@usp.br 


\begin{abstract}
:
Introduction: continuing education is a necessary personal and professional investment throughout the professional life of nurses as it expands and perfects technical, scientific, and practical skills. Objective: to describe the participation of nurses from the Americas region in the courses offered by the Virtual Campus of Public Health before and at the beginning of the COVID-19 pandemic, according to gender, age, educational level, and workplace. Method: a quantitative descriptive study. Data from the participants of the courses from January 2013 to June 2020 of the Virtual Campus of Public Health were analyzed. Study population: nurses from the Americas Region who took virtual courses. Descriptive statistics measures and proportions of the sociodemographic variables of the participants were calculated confidentially with authorization and credentials to access data on the platform. Results: 368,018 nurses were enrolled in the 515 virtual courses offered by the Virtual Campus of Public Health. The percentages of certified nurses according to the country of origin are as follows: Ecuador with $64.7 \%$, Mexico with $58.3 \%$, Honduras with $52.9 \%$, Paraguay with 48.6\%, Colombia with 45.2\%, Uruguay with 42.2\%, Argentina with 38.0\%, Chile with $22.7 \%$, Peru with $21.3 \%$, and Brazil with 9.7\%. 83.1\% were women, $75 \%$ had some degree of university education, $40 \%$ were between 26 and 35 years old, and $47.1 \%$ worked in hospitals. Conclusion: the COVID-19 pandemic reinforces the importance of virtual continuing education in which nurses can improve their knowledge, enhance their skills, and qualify for changes in practice, teaching, and research.
\end{abstract}

Keywords: nursing, nursing education, distance education, continuing education.

\title{
Resumo:
}

Introdução: a educação permanente é um investimento pessoal e profissional necessário ao longo da vida profissional do enfermeiro, pois amplia e aperfeiçoa as competências técnicas, científicas e práticas. Objetivo: descrever a participação de enfermeiros da Região das Américas nos cursos oferecidos pelo Campus Virtual de Saúde Pública antes e no início da pandemia COVID-19, segundo gênero, idade, escolaridade e local de trabalho. Método: estudo descritivo quantitativo. Foram analisados os dados dos participantes dos cursos de janeiro de 2013 a junho de 2020 do Campus Virtual de Saúde Pública. População do estudo: enfermeiros da Região das Américas que fizeram cursos virtuais. As medidas estatísticas descritivas e proporções das variáveis sociodemográficas dos participantes foram calculadas de forma confidencial com autorização e credencial para acesso aos dados na plataforma. Resultados: dos 515 cursos virtuais oferecidos pelo Campus Virtual de Saúde Pública, foram cadastrados 368.018 enfermeiros. As percentagens de enfermeiros certificados segundo o país de origem são as seguintes: Equador com 64,7\%, México com 58,3\%, Honduras com 52,9\%, Paraguai com 48,6\%, Colômbia com 45,2\%, Uruguai com 42,2\%, Argentina com 38,0\%, Chile com 22,7\%, Peru com $21,3 \%$ e Brasil com 9,7\%. 83,1\% eram mulheres, $75 \%$ possuíam ensino superior completo, $40 \%$ tinham entre 26 e 35 anos e $47,1 \%$ trabalhavam em hospitais. Conclusão: a pandemia COVID-19 reforça a importância da educação permanente virtual em que os enfermeiros podem melhorar seus conhecimentos, aprimorar suas habilidades e se qualificar para mudanças na prática, no ensino e na pesquisa.

Palavras-chave: enfermagem, educação em enfermagem, educação a distância, educação continuada.

\section{Introducción}

La pandemia de la COVID-19 ha puesto a prueba los sistemas de salud por el número de personas infectadas que requieren atención hospitalaria y también ha promovido la discusión mundial sobre la situación del trabajo, la formación y el número de los recursos humanos de salud disponibles en los países (1). También ha mostrado la necesidad e importancia de disponer de profesionales de salud en una cantidad adecuada a las necesidades de los sistemas de salud, que estén bien distribuidos y con buenas condiciones de trabajo, inclusive que tengan disponibilidad de equipos de protección individual y otros recursos como el apoyo al trabajo en equipo, capacitación y educación continua (2).

La educación continua es una inversión personal y profesional necesaria a lo largo de la vida profesional porque amplía y perfecciona los conocimientos técnicos y científicos, y las habilidades prácticas. Así mismo, permite la actualización en la formación posterior a la graduación profesional o de pregrado.

Frente a este contexto de fortalecer los recursos humanos para la salud en la región, la Organización Panamericana de Salud (OPS) y la Organización Mundial de Salud (OMS) requieren que los países desarrollen políticas de educación permanente sobre los recursos humanos para la salud, diversificando las metodologías, incorporando la educación virtual e innovando en el uso de las tecnologías para acompañar 
los procesos de cambio hacia el acceso universal a la salud (3). En la perspectiva de la educación continua de los recursos humanos para la salud, y específicamente de enfermería, se puede evidenciar el rol que tiene el Campus Virtual de Salud Pública (CVSP) (4).

Los cursos CVSP (5) son gratuitos y presentan dos formatos: autoaprendizaje y con tutoría. Las aulas ofrecen tres modalidades: autoaprendizaje (Massive Open Online Course, MOOC), países y regional. Los cursos en línea abiertos masivos (MOOC) son a los que se puede acceder a través de la web y generalmente no hay límites de participantes. Los cursos ofrecidos a nivel países o regional son de autoaprendizaje o con tutor.

Este artículo tiene como objetivos describir la participación de enfermeros de la Región de las Américas en los cursos ofrecidos por el CVSP, antes y al comienzo de la pandemia de la COVID-19, según su país, género, edad, nivel educativo y lugar de trabajo, y proponer sugerencias a partir de los datos analizados.

\section{Métodos}

Estudio de tipo descriptivo con enfoque cuantitativo. Fueron incluidos todos los enfermeros participantes en alguno de los cursos ofrecidos por la plataforma del CVSP durante el periodo del 2013 a junio del 2020. No se realiza cálculo de muestra porque se incluyen en el análisis todos los enfermeros que participaron en los cursos. Los criterios de inclusión de este curso son ser enfermero y estar inscrito en la plataforma durante el periodo de análisis y no fue incluido el personal de enfermería no profesional.

La información fue manejada de manera confidencial, y el CVSP autorizó y proporcionó un enlace y credenciales para acceder a los datos en la plataforma, con excepción de la información de identificación de los matriculados. Por esta razón, no se requirió una revisión por el consejo ético. La población de análisis fueron enfermeros de los países de la Región de las Américas que tomaron los cursos del CVSP.

Para fines de análisis de los cursos, fueron elegidos solamente los cincuenta cursos con más enfermeros matriculados. Para el análisis de datos se utilizó el programa Microsoft Excel y se calcularon las medidas de estadística descriptiva. Se reportó la distribución de frecuencias y proporciones para facilitar la codificación y tabulación.

\section{Aspectos éticos}

Este trabajo utilizó datos anonimizados. La información fue manejada de manera confidencial, y fue suministrada por la plataforma de CVSP de la OPS. Este trabajo cumple con los lineamientos éticos y de manejo de información que plantean las normas internacionales.

\section{Resultados}

El banco de datos reporta 464.493 matrículas de enfermeros en el CVSP desde el 2013 hasta fines de junio del 2020, de los cuales 2695 fueron excluidos ya que provenían de países fuera de la Región de las Américas y 20 de países que no son miembros o socios de la OPS como Anguila, Bonaire, Monserrat y Turcas y Caicos. Del restante de 461.778, fueron excluidos 26.560 profesionales que no eran del equipo de enfermería, resultando en 435.218. De estos, 367.998 eran enfermeros, 43.221 técnicos de enfermería y 23.999 auxiliares de enfermería. Dado que la participación de enfermeros era del $84,5 \%$, se optó por seguir el análisis solamente con este grupo profesional.

Del total de 367.998 enfermeros matriculados, 276.019 estaban matriculados en los cursos titulados MOOC, 38.630 en cursos ofertados directamente por los países y 53.349 en cursos ofertados a nivel regional por la sede de la OPS en Washington. La tabla 1 presenta esa distribución. 
TABLA 1.

\begin{tabular}{lrrr}
\hline \multirow{2}{*}{ Aula/Tipo de curso } & \multicolumn{1}{c}{ Matriculados } & \multicolumn{2}{c}{ Certificados } \\
\cline { 2 - 5 } MOOC & n & n & $\%$ \\
\hline Autoaprendizaje & & & \\
\hline Países & 276.019 & 139.148 & 50,4 \\
\hline Autoaprendizaje & & & \\
\hline Con tutoría & 12.641 & 14.308 & 53,3 \\
\hline Total & 25.989 & 21.051 & 54,5 \\
\hline Regional & 38.630 & & \\
\hline Autoaprendizaje & & 32.413 & 62,2 \\
\hline Con tutoría & 52.117 & 490 & 39,8 \\
\hline Total & 1232 & 32.903 & 61,7 \\
\hline Total general & 53.349 & 193.102 & 52,5 \\
\hline
\end{tabular}

Distribución de enfermeros matriculados y certificados por modalidad de curso Fuente: elaboración propia, datos provenientes de la plataforma CVSP

TABLA 2.

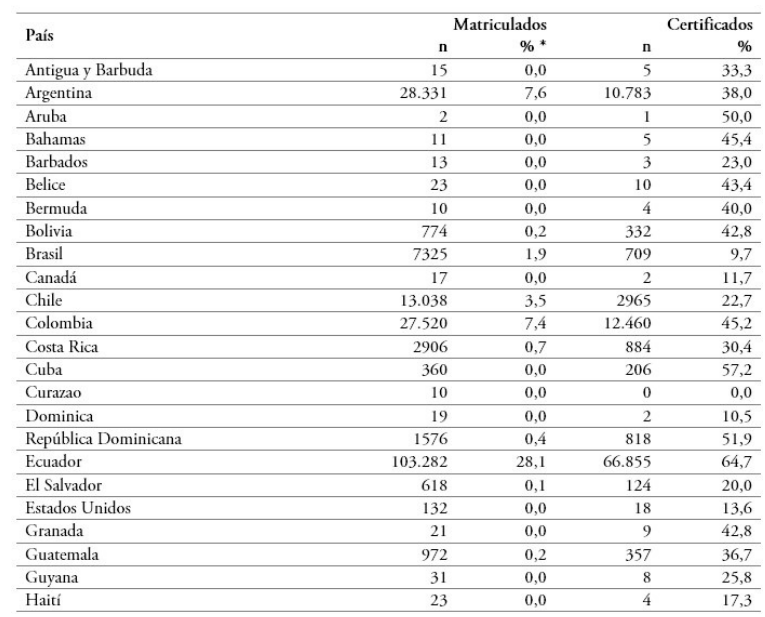

Distribución de matriculados y certificados por país

Fuente: elaboración propia, datos provenientes de la plataforma CVSP

La tabla 2 muestra que, de los matriculados por países, 145.786 (39,6\%) eran de México y $103.282(28,1 \%)$ de Ecuador. Los dos países representan 67\% de los matriculados en los cursos del CVSP. Ecuador es el país que tiene mayor número de enfermeros certificados (64,7\%), seguido por México (58,3\%), Cuba (57,2\%), Honduras (52,9\%) y Paraguay (48,7\%).

La tabla 3 muestra características de los enfermeros participantes de los cursos, la mayoría tiene entre 26 y 45 años $(63,7 \%)$.

TABLA 3.

\begin{tabular}{lrrrr}
\hline \multirow{2}{*}{ País } & \multicolumn{2}{c}{ Matriculados } & \multicolumn{2}{r}{ Certificados } \\
& $\mathbf{n}$ & $\boldsymbol{\%}^{*}$ & $\mathbf{n}$ & $\mathbf{\%}$ \\
\hline Honduras & 4870 & 1,3 & 2577 & 52,9 \\
\hline Jamaica & 29 & 0,0 & 12 & 41,3 \\
\hline México & 145.786 & 39,6 & 85.056 & 58,3 \\
\hline Nicaragua & 741 & 0,2 & 233 & 31,4 \\
\hline Panamá & 1624 & 0,4 & 615 & 37,8 \\
\hline Paraguay & 4346 & 1,1 & 2116 & 48,6 \\
\hline Perú & 18.136 & 4,9 & 3865 & 21,3 \\
\hline Puerto Rico & 368 & 0,1 & 103 & 27,9 \\
\hline San Cristóbal y Nieves & 27 & 0,0 & 12 & 44,4 \\
\hline Santa Luća & 144 & 0,0 & 43 & 29,9 \\
\hline San Vicente y las Granadinas & 31 & 0,0 & 14 & 45,1 \\
\hline Isla de San Martín & 1 & 0,0 & 0 & 0,0 \\
\hline Surinam & 18 & 0,0 & 9 & 50,0 \\
\hline Trinidad y Tobago & 78 & 0,0 & 12 & 15,3 \\
\hline Uruguay & 3697 & 1,0 & 1563 & 42,2 \\
\hline Venezuela & 1073 & 0,2 & 308 & 28,7 \\
\hline Total & 367.998 & $\mathbf{1 0 0 , 0}$ & 193.102 & 52,4 \\
\hline
\end{tabular}

Características de los enfermeros participantes de cursos disponibles en el Campus Virtual de Salud Pública Fuente: elaboración propia, datos provenientes de la plataforma CVSP

Los enfermeros con nivel universitario son 276.034 (75\%), 26.345 (7,2\%) tienen maestría y 1769 (0,5\%) doctorado. Los demás matriculados tienen otro nivel educativo. En relación con el lugar donde trabajan, 
173.327 (47,1\%) están en el hospital, 78.014 (21,2\%) en el Ministerio de Salud, 64.767 (17,6\%) en centros de salud y 35.695 (9,7\%) en universidades. Así, en el perfil de los enfermeros matriculados en los cursos disponibles en el CVSP están aquellos entre los 26 y 45 años que tienen solamente el grado universitario y que trabajan en hospitales.

La tabla 4 presenta los principales cursos en los cuales los enfermeros se matricularon y obtuvieron certificados. Los cursos en los que hubo un mayor número de enfermeros certificados en relación con los que se matricularon fueron: 1) Curso Virtual de Cólera; 2) Profilaxis Posexposición de Rabia; 3) Zika: Enfoque Clínico en la Atención Básica; 4) Interculturalidad y Género en el Marco de los Derechos Humanos; y 5) Curso Virtual Básico sobre Derechos Humanos.

El curso Virus Respiratorios Emergentes, Incluido el COVID-19: Métodos de Detección, Prevención, Respuesta y Control fue el más buscado con 49.406 matriculados; sin embargo, solamente 43,7\% de los matriculados concluyeron el curso con certificados de aprobación. Las razones por las que los enfermeros no finalizan el curso pueden ser dificultades con el internet, falta de tiempo disponible o conocimiento y falta de reconocimiento profesional por el curso realizado.

El curso con mayor acceso en países en la modalidad autoaprendizaje fue Interculturalidad y Género en el Marco de los Derechos Humanos, ofrecido en México en el 2020, el cual presentó 3532 matriculados y $73,2 \%$ con certificados de aprobación.

TABLA 4.

Principales cursos en que los enfermeros fueron matriculados y certificados

\begin{tabular}{lrr}
\hline Características de los enfermeros & n & $\%$ \\
\hline Género & & \\
\hline Femenino & 606.035 & 83,1 \\
\hline Masculino & & \\
\hline Edad (rango) & 67.694 & 18,4 \\
\hline $18-25$ & 146.860 & 40 \\
\hline $26-35$ & 87.234 & 23,7 \\
\hline $36-45$ & 47.720 & 13 \\
\hline $46-55$ & 15.208 & 4 \\
\hline $56-65$ & 2082 & 0,6 \\
\hline$>65$ & 1200 & 0,3 \\
\hline Sin dato & & \\
\hline Nivel educativo & 1769 & 0,4 \\
\hline Doctorado & 26.345 & 7 \\
\hline Maestría & 9 & 0 \\
\hline Especialización & 276.034 & 75 \\
\hline Universitario & 50.123 & 13,6 \\
\hline Otro & 13.718 & 4 \\
\hline Sin dato & & \\
\hline Lugar de trabajo & 78.014 & 21,2 \\
\hline Ministerio de Salud & 35.695 & 9,7 \\
\hline Universidad & 173.327 & 47,1 \\
\hline Hospital & 64.767 & 17,6 \\
\hline Centro de salud & 13.250 & 3,6 \\
\hline Sin dato & 2945 & 0,8 \\
\hline No trabaja & 367.998 & 100 \\
\hline Total & &
\end{tabular}

Fuente: elaboración propia, datos provenientes de la plataforma CVSP

La tabla 5 presenta los cursos de selección de los enfermeros, agrupados por categorías temáticas. Los cursos con mayor participación fueron: 1) Virus Respiratorios Emergentes, Incluido el COVID-19: Métodos de Detección, Prevención, Respuesta y Control 2) Curso Virtual sobre la Implementación del Paquete Técnico Hearts en la Atención Primaria de Salud; 3) Prevención y Control de Infecciones (PCI) Causadas por el Nuevo Coronavirus (COVID-19); 4) Liderazgo en Enfermería: Empoderamiento de los Enfermeros Líderes en Latinoamérica; 5) ePROTECT Infecciones respiratorias: Salud y seguridad ocupacional.

$\mathrm{Al}$ categorizar los cursos del CVSP por temas y aulas, se evidencia que los relacionados a enfermedades y vigilancia epidemiológica (80,9\%), vacunación (80,8\%), salud mental-prevención de la autolesión y suicidio: 
empoderamiento de los profesionales de atención primaria de salud $(69,1 \%)$ e infecciones respiratorias $(68,1 \%)$ fueron los que más terminaron los matriculados. Los cursos que recibieron más matriculas fueron los relacionados al tema COVID-19, liderazgo en enfermería, Hearts, infecciones respiratorias y cáncer cervicouterino. Los enfermeros mostraron gran interés en los cursos sobre enfermedades de vigilancia epidemiológica en aulas MOOC en los ámbitos país y regional, como Capacitación en Vigilancia en Salud.

Así mismo, los cursos con más enfermeros matriculados fueron: Curso Virtual sobre la Implementación del Paquete Técnico Hearts en la Atención Primaria de la Salud, Prevención y Control de Infecciones Causadas por el Nuevo Coronavirus, Liderazgo en Enfermería, y Manejo de Hipertensión Arterial para Equipos de APS. En el aula regional, en la modalidad autoaprendizaje, el curso con mayor acceso fue Promoción de la Salud.

TABLA 5.

\begin{tabular}{|c|c|c|c|}
\hline \multirow{2}{*}{ Posición/curso } & \multirow{2}{*}{ Matriculados } & \multicolumn{2}{|c|}{ Certificados } \\
\hline & & n & \\
\hline $\begin{array}{l}\text { 1. Virus Respiratorios Emergentes, Incluido el COVID-19: Métodos de Detección, } \\
\text { Prevención, Respuesta y Control }\end{array}$ & 49.406 & 21.591 & 43,7 \\
\hline $\begin{array}{l}\text { 2. Curso Virtual sobre la Implementación del Paquete Técnico Hearts en la Atención } \\
\text { Primaria de Salud }\end{array}$ & 29.457 & 13.894 & 47,2 \\
\hline 3. Prevención y Control de la COVID-19 & 25.226 & 16.152 & 64 \\
\hline 4. Liderazgo en Enfermería: Empoderamiento de los Enfermeros Líderes en Latinoamérica & 20.423 & 10.179 & 49,8 \\
\hline 5. ePROTECT Infecciones Respiratorias: Salud y Seguridad Ocupacional & 16.415 & 12.090 & 73,7 \\
\hline 6. Curso Virrual en Control Integral del Cáncer Cervicouterino & 8860 & 2216 & 25 \\
\hline 7. Promoción de la Salud & 8423 & 5240 & 62,2 \\
\hline 8. Cadena de Frío para Inmunización Segura & 7361 & 6515 & 88,5 \\
\hline $\begin{array}{l}\text { 9. Prevención Secundaria de Enfermedades Cardiovasculares para el Equipo del Primer Nivel } \\
\text { de Atención }\end{array}$ & 7096 & 3314 & 46,7 \\
\hline 10. Zika: Enfoque clínico en la Atención Básica & 5795 & 4383 & 75,6 \\
\hline 11. Curso de Manejo Clínico de las Infecciones Respiratorias Agudas Graves - IRAG-2020 & 5773 & 3209 & 55,6 \\
\hline 12. Curso Virtual sobre el Correcto Llenado del Cerrificado de Defunción, RELACSIS & 5484 & 3616 & 65,9 \\
\hline 13. Curso Virtual de Cólera & 3877 & 3490 & 90 \\
\hline 14. Virus Respiratorios Emergentes, COVID-19 & 3783 & 130 & 3,4 \\
\hline 15. Acceso y Uso de la Información Científica en Salud & 3775 & 2584 & 68,5 \\
\hline 16. Profilaxis Posexposición de la Rabia & 3640 & 2809 & 77,2 \\
\hline 17. Curso Virtual Rásico sobre Derechos Humanos y Salud & 3629 & 2623 & 72,3 \\
\hline 18. Interculturalidad y Género en el Marco de los Derechos Humanos-2020 & 3532 & 2584 & 73,2 \\
\hline 19. Primera Ayuda Psicológica & 3056 & 1769 & 57,9 \\
\hline 20. Otros cursos & 152.987 & 74.714 & 48,5 \\
\hline Total & 367.998 & 193.102 & 52,5 \\
\hline
\end{tabular}

Cursos del CVSP categorizados por temas y aulas Fuente: elaboración propia, datos provenientes de la plataforma CVSP

\section{Discusión}

A nivel mundial, la fuerza laboral de enfermería es relativamente joven: el $38 \%$ de las enfermeras tiene menos de 35 años, en comparación con el 17\% que tiene 55 años y más, pero hay disparidades entre regiones, con estructuras de edad sustancialmente mayores en las Américas (6).

Los mundos del trabajo y la educación se entrelazan en el ámbito de la formación profesional en enfermería con diferentes regulaciones, reglamentaciones, intereses y prácticas, y, sobre todo, con sus conceptos y referencias teóricas subyacentes. El conocimiento científico y la tecnología, como matrices de desarrollo, imponen modelos y parámetros a las políticas públicas, sin que estas hayan superado las antiguas formas de exclusión social y la perspectiva económica de la dependencia, cuyas repercusiones se han potenciado dada la relevancia del nuevo coronavirus (7).

Se evidencia en los resultados que se requiere aumentar la oferta de cursos en temas según las necesidades educacionales que presentan los enfermeros, aumentar la participación de enfermeros de Brasil, Canadá, el Caribe inglés y de Estados Unidos. Dado que los cursos tienen una audiencia global, es recomendable que el contenido se adapte a los diferentes contextos culturales y niveles de los participantes (8). Además, es necesario mayor divulgación de los cursos para el personal que trabaja en el primer nivel de atención para lograr mayor acceso.

La literatura categoriza a los estudiantes de cursos virtuales como comprometidos (personas que participaron en todas las evaluaciones hasta el final del curso), estudiantes que abandonaron temprano (personas que inicialmente participaron pero no terminaron el curso) y otros estudiantes (el resto) (9). Los cursos MOOC tienen tasas de finalización relativamente bajas, con un promedio del 22\% (10). Sin embargo, 
se puede considerar que los cursos del CVSP tienen un buen resultado ya que alrededor del $50 \%$ de los enfermeros matriculados llegan a terminarlos.

Los datos están en conformidad con la afirmación de que la educación permanente debe dirigirse a reducir las brechas de conocimiento y permitir la construcción de un sistema de aprendizaje que apoye el desarrollo de habilidades, y promueva las competencias técnicas, programáticas, gerenciales y administrativas (11). Es muy importante la creación de mecanismos que impidan la evasión de los estudiantes y se sugiere que el CVSP busque estrategias para evaluar las causas del abandono de participantes cuando esto sucede.

\section{Conclusiones}

Las estrategias educativas, principalmente de recursos virtuales, se han convertido en una herramienta útil, posibilitando el acceso al conocimiento y la superación de barreras geográficas y temporales. En el tiempo de la pandemia de la COVID-19, los recursos virtuales se han convertido en una importante fuente de conocimientos y de intercambio de experiencias para todos, especialmente para los profesionales de salud. La actual pandemia refuerza la importancia de la educación continua virtual en la que los enfermeros pueden mejorar sus conocimientos, potencializar sus habilidades, y calificarse para los cambios en la práctica, la enseñanza y la investigación.

La experiencia relatada del CVSP y de la búsqueda de los enfermeros de la Región de las Américas de la educación continua puede ser un aporte importante en el ámbito global.

Limitaciones: los datos descriptivos y retrospectivos sobre la información del registro de los estudiantes no permiten hacer un análisis que explore otras variables como las dificultades de finalización de los cursos, la disponibilidad de infraestructura y recursos virtuales, y las razones de mayor participación en algunas regiones o zonas determinadas, entre otras.

\section{Financiamiento}

Ninguno

\section{Conflicto de intereses}

Los autores declaran no tener conflicto de intereses

\section{Referencias}

1. Benjamin GC. Ensuring health equity during the COVID-19 pandemic: the role of public health infrastructure. Rev Panam Salud Publica. 2020;44:e70. https://doi.org/10.26633/RPSP.2020.70

2. Cassiani SHB, Munar Jimenez EF, Umpiérrez Ferreira A, Peduzzi M, Leija Hernández C. La situación de la enfermería en el mundo y la Región de las Américas en tiempos de la pandemia de COVID-19. Rev Panam Salud Pública. 2020;44: e64. https://doi.org/10.26633/RPSP.2020.64

3. Organización Panamericana de la Salud. Plan de acción sobre recursos humanos para el acceso universal a la salud y la cobertura universal de salud 2018-2023 [Internet]. 56. Consejo directivo 70a sesión del comité Regional de la OMS para las Américas: 31 de agosto 2018 [citado 10 Jun 2020]. Washington, DC: OPS; 2018. Disponible en: https://www.paho.org/ 
4. De Bortoli-Cassiani SH, Antonio-Costa L, Hoyos MC, De Gracia E, Listovsky G, Menezes-da Silva FA. Participación de enfermeros(as) de la Región de las Américas en una capacitación virtual sobre liderazgo. Enfermería Universitaria. 2019 Sep; 16(3): 230-9. http://dx.doi.org/10.22201/eneo.23958421e.2019.3.688

5. Organización Panamericana de Salud. Campus virtual de salud pública ¿Qué es el campus? [Internet]; 2020 [citado 30 Jun 2020]. Disponible en: https://www.campusvirtualsp.org/es/que-es-el-campus

6. Ortega J, Hooshmand M, Foronda C, Padron M, Simon D, et al. Developing nurse leaders across the Americas: evaluation of an online nursing leadership course. Rev Panam Salud Publica. 2018;42:e152. https://doi.org/10 $.26633 /$ RPSP.2018.152

7. World Health Organization. State of the world's nursing 2020: investing in education, jobs and leadership [Internet]. Geneva: WHO; 2020 [citado 5 Jul 2020]. Disponible en: https://apps.who.int/iris/handle/10665/331677

8. Costa R, Lino MM, Souza AIJ, Lorenzini E, Fernandes GCM, Brehmer LCF, et al. Enseñanza de enfermería en tiempos de COVID-19: ¿cómo reinventarla en este contexto? Texto Contexto Enferm. 2020;29:e20200202. ht tps://doi.org/10.1590/1980-265X-TCE-2020-0002-0002

9. Culquichicón C, Helguero-Santin LM, Labán-Seminario LM, Cardona-Ospina JA, Aboshady OA, Correa R. Massive open online courses in health sciences from Latin American institutions: A need for improvement? F1000Research. 2017;6:940. http://dx.doi:10.12688/f1000research.11626.1

10. Jordan, Katy (2015). Massive open online course completion rates revisited: Assessment, length and attrition. IRRODL. 2015;16(3):341-58. http://dx.doi.org/10.13140/RG.2.1.2119.6963

11. Cho M, Moya J, Hernández T, Flores M, Ledezma Y, Samán L, Listovsky G. Desarrollo del nodo Venezuela en el campus virtual de la salud pública. Rev Panam Salud Publica. 2018;42:e71. https://doi. org/10.26633/ RPSP.2018.71

\section{Notas}

* Artículo de investigación.

Licencia Creative Commons CC BY 4.0

Cómo citar este artículo: Cassiani S, De Almeida RT, Hoyos Garcia MC, Listovsky G, De Gracia Tejada EM, Saldarriaga Sandoval LJ, Menezes da Silva FA. Educación continua en enfermería: Campus Virtual en Salud Pública en la Región de las Américas. Investig Enferm Imagen Desarr. 2021;23. https://doi.org/10.11144/ Javeriana.ie23.ecec. 